\title{
Seasonal dynamics of canine antibody response to Phlebotomus perniciosus saliva in an endemic area of Leishmania infantum
}

Rita Velez ${ }^{1,2^{*}}$, Tatiana Spitzova ${ }^{3}$, Ester Domenech ${ }^{4}$, Laura Willen ${ }^{3}$, Jordi Cairó ${ }^{4}$, Petr Volf ${ }^{3}$ and Montserrat Gállego ${ }^{1,2^{*}}$

\begin{abstract}
Background: Canine leishmaniosis (CanL) is an important zoonotic parasitic disease, endemic in the Mediterranean basin. In this region, transmission of Leishmania infantum, the etiological agent of CanL, is through the bite of phlebotomine sand flies. Therefore, monitoring host-vector contact represents an important epidemiological tool, and could be used to assess the effectiveness of vector-control programmes in endemic areas. Previous studies have shown that canine antibodies against the saliva of phlebotomine sand flies are specific markers of exposure to Leishmania vectors. However, this method needs to be further validated in natural heterogeneous dog populations living in CanL endemic areas.

Methods: In this study, 176 dogs living in 12 different locations of an L. infantum endemic area in north-east Spain were followed for 14 months. Blood samples were taken at 5 pre-determined time points (February, August and October 2016; January and April 2017) to assess the canine humoral immune response to whole salivary gland homogenate $(\mathrm{SGH})$ and to the single salivary $43 \mathrm{kDa}$ yellow-related recombinant protein (rSPO3B) of Phlebotomus perniciosus, a proven vector of $L$. infantum naturally present in this region. Simultaneously, in all dogs, L. infantum infection status was assessed by serology. The relationship between anti-SGH and anti-rSP03B antibodies with the sampling month, L. infantum infection and the location was tested by fitting multilevel linear regression models.

Results: The dynamics of canine anti-saliva IgG for both SGH and rSPO3B followed the expected trends of $P$. perniciosus activity in the region. Statistically significant associations were detected for both salivary antigens between vector exposure and sampling month or dog seropositivity to L. infantum. The correlation between canine antibodies against SGH and rSP03B was moderate.

Conclusions: Our results confirm the frequent presence of CanL vectors in the study area in Spain and support the applicability of SGH- and rSP03B-based ELISA tests to study canine exposure to P. perniciosus in L. infantum endemic areas.
\end{abstract}

Keywords: Canine leishmaniosis, Phlebotomus perniciosus, Saliva proteins, Markers of exposure, Longitudinal study, Northeast Spain

\section{Background}

Leishmania infantum (Kinetoplastida: Trypanosomatidae) is the causative agent of canine leishmaniosis (CanL), a zoonotic vector-transmitted disease widespread in the Mediterranean region, as well as in other parts of the world [1-3]. Prevalence of L. infantum infection in canine populations from endemic areas is highly heterogeneous [4], and not all infected dogs will ever develop

\footnotetext{
* Correspondence: rita.velez@isglobal.org; mgallego@ub.edu

${ }^{1}$ ISGlobal, Hospital Clínic - Universitat de Barcelona, Barcelona, Spain Full list of author information is available at the end of the article
}

clinical signs of the disease [5]. However, infected asymptomatic dogs could act as a reservoir of the parasite and are capable of transmitting L. infantum to other dogs, as well as to humans $[6,7]$.

The transmission of the parasite is mainly vectorial, through the bite of phlebotomine sand flies. In the Mediterranean basin, eight species of the genus Phlebotomus have been implicated as vectors of $L$. infantum, according to conventional criteria. From these, all except one belong to the subgenus Larroussius [8]. In Spain, CanL transmission is mainly shared by $P$. (L.) perniciosus and $P$. (L.) 
ariasi $[9,10]$, with the second species having a narrower distribution but being responsible for maintaining the infection at higher altitudes $[11,12]$. Recently, $L$. infantum DNA was also found in another Larroussius species, $P$. langeroni, in the south of the country [13].

The detection of anti-sand fly salivary antibodies in the blood of vertebrate hosts has proven to be highly specific [14] and was successfully used as a marker of exposure to L. infantum vectors $[15,16]$. In CanL endemic areas, monitoring the canine IgG response to sand fly saliva can be a useful epidemiological tool $[15,17]$, complementing studies of vector population dynamics and host-vector interactions, as well as enabling the assessment of risk of Leishmania infection [14, 18, 19]. Furthermore, it can be used to measure the effectiveness of vector-control programmes and to assist in the design of better control strategies for the disease [20, 21].

Originally, sand fly whole salivary gland homogenates (SGH) were used to investigate the presence of anti-sand fly saliva antibodies in vertebrate hosts [20-22]. However, its use in large-scale studies is impaired by technical limitations [23]. Additionally, the use of SGH in vector exposure tests may reduce the specificity of detection due to a possible cross-reactivity with saliva of sympatric and closely related sand fly species [24].

An alternative to the use of SGH is the identification of species-specific salivary proteins that can be expressed in recombinant forms and produced in large quantities for use in large-scale epidemiological studies [25, 26]. Recent studies identified $P$. perniciosus yellow-related protein rSP03B as the most promising candidate to replace SGH in the detection of host markers of exposure to this vector species $[16,17,26]$. This recombinant protein has been tested and validated in dogs and other animals in cross-sectional studies $[16,26]$, as well as in a canine longitudinal study [17], but no information exists on the seasonal dynamics of either SGH or rSP03B in natural heterogeneous dog populations from endemic areas.

Therefore, the objectives of this study were (i) to investigate the dynamics of $P$. perniciosus and their relative density in a previously uncharacterized CanL endemic area through the detection of anti-saliva IgG in dogs; and (ii) to evaluate the performance of both SGH and rSP03B antigens as markers of exposure to $P$. perniciosus in natural canine populations.

\section{Results}

Seasonal dynamics of IgG response against salivary proteins from $P$. perniciosus

Median values of normalized ELISA OD values for SGH ranged from 9.04 (range: 3.94-66.23) in January 2017 to 18.51 (7.93-100.58) in August 2016 (Table 1). For rSP03B, median OD values varied between $12.21(6.75-53.71)$ and 19.53 (10.64-124.01) in January 2017 and August 2016,
Table 1 Median values of normalized OD readings for SGH and rSP03B obtained per sampling month in all locations

\begin{tabular}{llll}
\hline Variable & $N$ & $\begin{array}{l}\text { SGH } \\
\text { Median (Range) }\end{array}$ & $\begin{array}{l}\text { rSP03B } \\
\text { Median (Range) }\end{array}$ \\
\hline February 2016 & 174 & $10.11(5.49-49.62)$ & $14.67(7.36-41.24)$ \\
August 2016 & 33 & $18.51(7.93-100.58)$ & $19.53(10.64-124.01)$ \\
October 2016 & 164 & $11.15(5.56-86.44)$ & $15.31(6.15-112.54)$ \\
January 2017 & 154 & $9.04(3.94-66.23)$ & $12.21(6.75-53.71)$ \\
April 2017 & 148 & $9.54(5.25-62.59)$ & $13.44(6.27-36.22)$ \\
\hline
\end{tabular}

Abbreviation: $N$ number of dogs sampled per sampling month

respectively. With both antigens, median OD readings raised from basal values in February 2016 (10.11 and 14.67 for SGH and rSP03B, respectively) to peak in August (18.51 and 19.53 for SGH and rSP03B, respectively), sustained higher readings in October (11.15 and 15.31 for SGH and rSP03B, respectively), and descended again to basal levels in January (9.04 and 12.21 for SGH and rSP03B, respectively) and April 2017 (9.54 and 13.44 for SGH and rSP03B, respectively). Median normalized ELISA OD results obtained per month for both SGH and rSP03B are described in Table 1 and plotted in Fig. 1.

Cut-off values were set at 13 for SGH and 22 for rSP03B. When these were applied to the OD readings obtained in August 2016, 75.76\% (25/33) of the dogs were positive to anti-SGH IgG, and $36.36 \%(12 / 33)$ to anti-rSP03B antibodies. In October, these values dropped to $35.98 \%$ (59/ 164) for SGH and $18.9 \%$ (31/164) for rSP03B. During the non-transmission season (considered to extend from

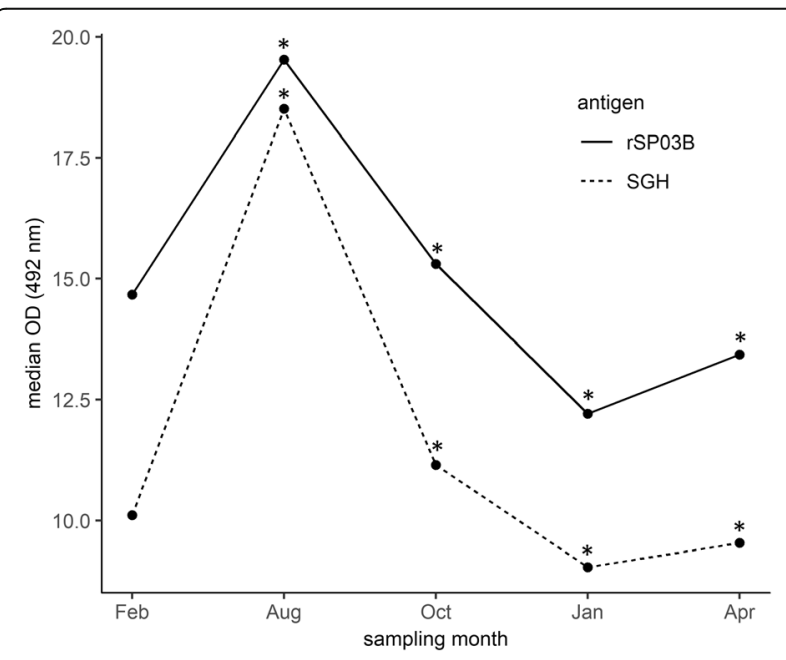

Fig. 1 Dynamics of anti-P. perniciosus salivary proteins IgG response in dogs from an endemic area during a sand fly activity season. Values presented refer to the normalized OD medians obtained at each sampling month for all dogs and locations. Statistically significant differences in median OD between two consecutive months are marked with an asterisk $(P<0.05)$ 
November to May), the percentage of seropositive dogs ranged from 14.29\% (25/175) in February 2016 to 17.57\% (26/148) in April 2017 for SGH and 8.44\% (13/154) in January 2017 to $12.16 \%$ (18/148) in April 2017 for rSP03B.

Correlation results for IgG response between SGH and rSP03B were $r_{S}=0.54$ (95\% CI: $0.48-0.60, P<0.001$ ) (Fig. 2).

\section{Dogs' exposure to $P$. perniciosus in the study area}

Exposure of dogs to phlebotomine vectors showed some variation according to the location. Median OD readings varied from 9.11 (range: 5.25-20.57) to 14.14 (7.44-55.45) for SGH ELISA and from 12.71 (7.53-64.44) to 17.87 (8.39-112.54) for rSP03B. Minimum median values of response to both SGH and rSP03B corresponded to the same location (Aiguaviva), but maximum median values were registered in different sites for each antigen (Sant Feliu de Guíxols for SGH and Montagut for rSP03B) (Table 2). Figure 3 presents the dynamics of dogs' IgG response to SGH (Fig. 3a) and rSP03B (Fig. 3b) in each locality.

The percentage of anti-sand fly saliva seropositive dogs per location, defined as the number of dogs that showed a positive IgG titre at least once during the study period, ranged from $13.33 \%(1 / 8)$ in Ordis to $100 \%$ in Canet d'Adri (8/8) and Sant Feliu de Guíxols $(4 / 4)$ for SGH, and from $8.16 \%(1 / 12)$ in Hostalnou de Bianya to $100 \%(4 / 4)$ in Sant Feliu de Guíxols for rSP03B. Total anti-sand fly saliva seropositivity calculated for the study area was $49.43 \%$ (87/176) for

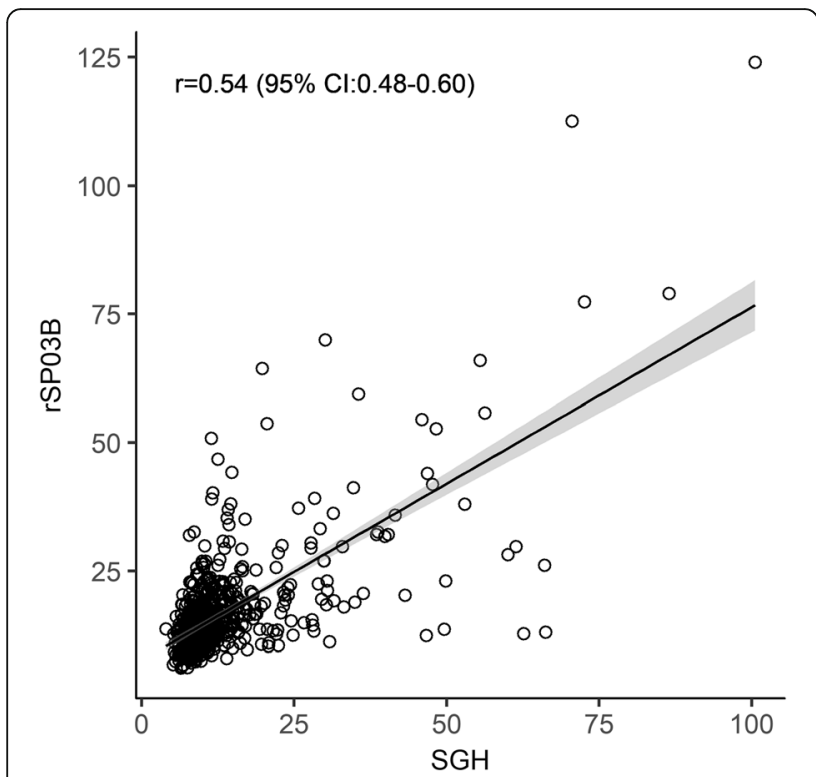

Fig. 2 Correlation between IgG recognizing SGH and rSP03B protein in dogs naturally exposed to $P$. perniciosus. Results from both $\mathrm{SGH}$ and rSP03B are presented in normalized OD $\left(r_{S}=0.54 ; 95 \%\right.$ Cl: $0.48-$ $0.60, P<0.001)$
anti-SGH IgG and $28.98 \%(51 / 176)$ for anti-rSP03B antibodies.

Dogs' exposure to $P$. perniciosus and $L$. infantum infection Correlation results between antibody response to $P$. perniciosus saliva and L. infantum were low both for SGH $\left(r_{S}=0.27,95 \% \mathrm{CI}: 0.19-0.35, P<0.001\right)$ and $\mathrm{rSP} 03 \mathrm{~B}$ protein $\left(r_{S}=0.25,95 \% \mathrm{CI}: 0.18-0.32, P<0.001\right)$.

Multilevel analysis of the relationship between anti- $P$. perniciosus salivary proteins, month and location and $L$. infantum seropositivity

The multilevel model results confirmed the annual dynamics of anti-salivary proteins IgG responses. When compared to the first sampling month (February 2016), IgG responses to SGH significantly rose in August $(t=8.55, d f=491, P<0.001)$ and October $(t=6.49, d f=$ 491, $P<0.001)$ and dropped in January $(t=-2.49, d f=$ 491, $P=0.013$ ) and April 2017 (no significant difference when compared to February 2016). As expected, the highest log OD estimate was observed in August 2016 and the lowest in January 2017 (Table 3). The same trend was observed in the model run for the rSP03B protein, with comparable levels of significance (Table 4). There were no significant differences in IgG responses for both antigens between each sampling location and the one set as reference, except for Montagut, where significantly higher OD levels were observed for SGH $(t=2.28, d f=166, P=$ $0.024)$ and $\mathrm{rSP} 03 \mathrm{~B}(t=2.13, d f=164, P=0.035)$. According to the multilevel model, seropositivity to L. infantum proved to be associated with a rise in anti-salivary proteins OD values for both SGH $(t=2.5, d f=491, P=0.013)$ and rSP03B $(t=2.15, d f=493, P=0.032)$.

\section{Discussion}

The quantification of anti-sand fly saliva antibodies in vertebrate hosts of $L$. infantum has been previously shown to be an effective way of measuring exposure to the parasite vectors [16]. In the case of dogs, the most frequent host and reservoir of $L$. infantum, this has been proven for $P$. perniciosus $[15,26]$, as well as for other sand fly species [27-29]. These markers of exposure can then be applied in host-vector epidemiological studies, in L. infantum infection risk assessment, and to assist in the design of control strategies for the disease. Therefore, it is important to validate these techniques in natural, heterogeneous populations from endemic areas, in which a higher individual variability is expected.

Phlebotomus perniciosus activity period in Spain shows two main peaks, the first in June-July and the second in September-October. These peaks also correspond to the periods of highest $L$. infantum transmission [30-32]. This trend was identified in our study and corresponds to the rise in anti-saliva antibody levels observed between 
Table 2 Median values of normalized OD readings for SGH and rSP03B obtained per sampling location at all time points

\begin{tabular}{|c|c|c|c|c|}
\hline \multirow[t]{2}{*}{ Variable } & \multirow[t]{2}{*}{$n$ (Range) } & \multirow{2}{*}{$\begin{array}{l}\text { Geographical } \\
\text { coordinates }\end{array}$} & $\mathrm{SGH}$ & \multirow{2}{*}{$\begin{array}{l}\text { rSP03B } \\
\text { Median (Range) }\end{array}$} \\
\hline & & & Median (Range) & \\
\hline Ordis & $8(7-9)$ & $42^{\circ} 13^{\prime} 37.7^{\prime \prime} \mathrm{N}, 2^{\circ} 54^{\prime} 24.1 \mathrm{E}$ & $9.14(6.45-45.95)$ & $15.16(8.35-54.50)$ \\
\hline Madremanya & $14(12-15)$ & $41^{\circ} 58^{\prime} 47.0^{\prime \prime N}, 2^{\circ} 58^{\prime} 7.2^{\prime \prime} \mathrm{E}$ & $11.22(6.79-49.84)$ & $14.49(8.95-43.99)$ \\
\hline Vidreres & $8(7-9)$ & $41^{\circ} 47^{\prime} 27.4^{\prime \prime} \mathrm{N}, 2^{\circ} 45^{\prime} 0.4^{\prime \prime} \mathrm{E}$ & $10.59(7.80-16.86)$ & $13.46(8.58-40.23)$ \\
\hline Massanes & $21(20-23)$ & $41^{\circ} 45^{\prime} 15.3^{\prime \prime} \mathrm{N}, 2^{\circ} 38^{\prime} 44.0^{\prime \prime} \mathrm{E}$ & $9.31(5.67-62.59)$ & $16.35(7.82-55.81)$ \\
\hline Hostalnou de Bianya & $12(11-14)$ & $42^{\circ} 13^{\prime} 26.0^{\prime \prime N}, 2^{\circ} 26^{\prime} 9.7^{\prime \prime} \mathrm{E}$ & $8.75(5.35-33.16)$ & $13.19(6.27-46.82)$ \\
\hline Montagut & $13(7-15)$ & $42^{\circ} 14^{\prime} 7.7^{\prime \prime} \mathrm{N}, 2^{\circ} 35^{\prime} 57.6^{\prime \prime} \mathrm{E}$ & $12.01(3.94-72.61)$ & $17.87(8.39-112.54)$ \\
\hline St. Esteve de Llémena & $9(9-10)$ & $42^{\circ} 3^{\prime} 35.1^{\prime \prime} \mathrm{N}, 2^{\circ} 37^{\prime} 1.4^{\prime \prime} \mathrm{E}$ & $9.49(6.23-22.40)$ & $14.18(9.12-22.46)$ \\
\hline Canet d'Adri & $8(4-10)$ & $42^{\circ} 1{ }^{\prime} 53.7^{\prime \prime} \mathrm{N}, 2^{\circ} 44^{\prime} 15.3^{\prime \prime} \mathrm{E}$ & $10.61(6.52-100.58)$ & $14.03(7.36-124.01)$ \\
\hline Aiguaviva & $19(16-22)$ & $41^{\circ} 54^{\prime} 27.2^{\prime \prime} \mathrm{N}, 2^{\circ} 46^{\prime} 19.0^{\prime \prime} \mathrm{E}$ & $9.11(5.25-20.57)$ & $12.71(7.53-64.44)$ \\
\hline St. Feliu de Guíxols & 4 & $41^{\circ} 47^{\prime} 2.3^{\prime \prime N}, 2^{\circ} 59^{\prime} 58.7^{\prime \prime E}$ & $14.14(7.44-55.45)$ & $16.73(8.57-65.97)$ \\
\hline Riells i Viabrea & $20(18-21)$ & $41^{\circ} 43^{\prime} 59^{\prime \prime} \mathrm{N}, 2^{\circ} 33^{\prime} 39.3^{\prime \prime E}$ & $10.02(6.07-66.23)$ & $13.43(8.59-35.31)$ \\
\hline Vilobí d'Onyar & $23(22-23)$ & $41^{\circ} 53^{\prime} 3.2^{\prime \prime} \mathrm{N}, 2^{\circ} 43^{\prime} 38.6^{\prime \prime} \mathrm{E}$ & $9.13(5.17-16.49)$ & $13.05(6.15-38.07)$ \\
\hline
\end{tabular}

Abbreviation: $n$ mean number of dogs sampled in each location

August and October. Humoral immune response to $P$. perniciosus saliva elicited in experimentally bitten dogs showed that antibody levels significantly rose after $2-4$ weeks of continued exposure, peaking in week 5 [15]. In our field study, the highest IgG levels were in August, which clearly corresponded to the June-July P. perniciosus expected activity peak. Similarly, the high IgG readings obtained in October are likely to correspond to P. perniciosus second peak of activity. The lower rise in antibody levels observed at this time point can be explained by an earlier sampling at the beginning of October, which may have hindered the display of a complete seroconversion. The high overall levels of seropositivity to anti-sand fly saliva antigens, especially for SGH (49.43\%), strongly support the CanL endemicity status for the region [33]. These results also validate both $\mathrm{SGH}$ and $\mathrm{rSP} 03 \mathrm{~B}$ as suitable antigens to assess exposure to $P$. perniciosus in natural canine populations from endemic areas.

An important remark when analysing the longitudinal dynamics of anti-sand fly saliva IgG in the study dog

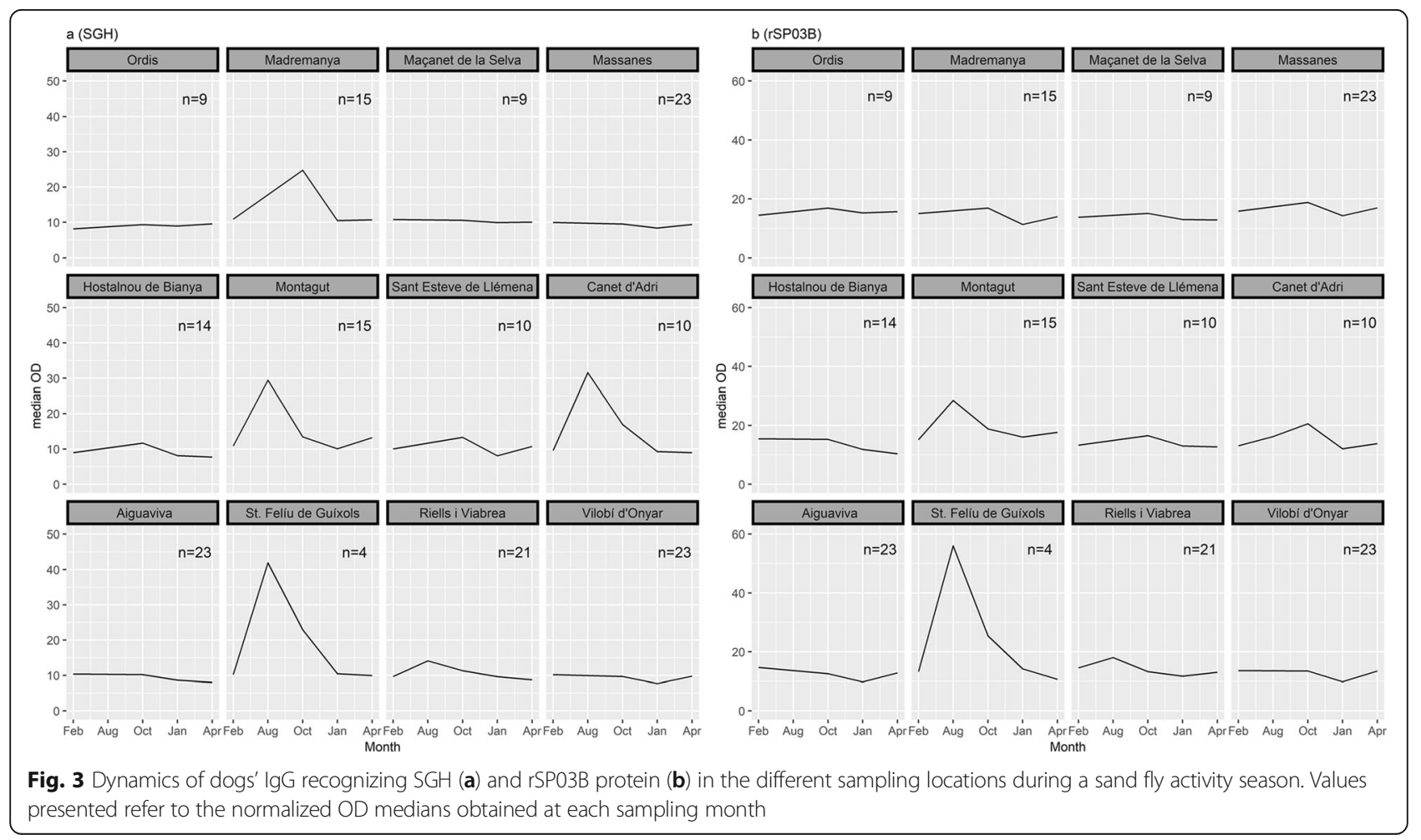


Table 3 Estimates of the multilevel linear regression model of the relationship between log transformed normalized SGH OD values and sampling time, location and dog seropositivity to $L$. infantum. "Dog" was included as a random effects variable

\begin{tabular}{lllll}
\hline Variable & Levels & Estimate & SE & $P$-value \\
\hline Intercept & & 2.40 & 0.06 & $<0.001$ \\
Sampling month & February 2016 & Ref & - & - \\
& August 2016 & 0.54 & 0.06 & $<0.001$ \\
& October 2016 & 0.20 & 0.03 & $<0.001$ \\
& January 2017 & -0.06 & 0.03 & 0.013 \\
& April 2017 & -0.01 & 0.03 & 0.666 \\
Location & Aiguaviva & Ref & - & - \\
& Ordis & 0.07 & 0.11 & 0.562 \\
& Madremanya & 0.08 & 0.10 & 0.427 \\
& Vidreres & 0.10 & 0.11 & 0.393 \\
& Massanes & 0.07 & 0.09 & 0.441 \\
& Hostalnou de Bianya & -0.08 & 0.10 & 0.404 \\
L. infantum & Montagut & 0.22 & 0.10 & 0.024 \\
& St. Esteve de Llémena & -0.03 & 0.11 & 0.786 \\
& Canet d'Adri & -0.02 & 0.11 & 0.891 \\
& St. Feliu de Guíxols & 0.16 & 0.15 & 0.308 \\
& Riells i Viabrea & 0.03 & 0.09 & 0.703 \\
& Vilobí d'Onyar & -0.02 & 0.09 & 0.791 \\
Seronegative & Ref & - & - \\
& Seropositive & 0.10 & 0.04 & 0.013 \\
\hline
\end{tabular}

Abbreviation: SE standard error

aLevel of significance of $P$-value $<0.05$ was used

population is that there was a clear basal antibody level before the transmission season. After the expected rise in humoral response during summer months, IgG levels returned again to basal levels. These results show that, though exposed to repetitive bites during several months, dogs from endemic areas do not sustain high anti-saliva IgG levels throughout the year, allowing the detection of recent exposure to sand flies in natural populations. Similar results were recently reported in a longitudinal field study in Brazil, where canine IgG against Lutzomyia longipalpis saliva were evaluated [34]. Our study identified the same trends for both $\mathrm{SGH}$ and rSP03B, which reinforces the suitability of recombinant antigens in detecting recent exposure to phlebotomine vectors in endemic settings, particularly when considering the use of these tests in large-scale studies for vector control interventions [35, 36].

Antibodies recognizing both SGH and rSP03B followed similar dynamics throughout the field study. However, the correlation between the two antigens was only moderate $\left(r_{S}=0.54 ; 95 \%\right.$ CI: $\left.0.48-0.60, P<0.001\right)$. Even so, available studies show that rSP03B is the most promising surrogate for $\mathrm{SGH}$ as a marker of exposure to P. perniciosus in the
Table 4 Estimates of the multilevel linear regression model of the relationship between log transformed normalized rSP03B OD values and sampling time, location and dog seropositivity to $L$. infantum. "Dog" was included as a random effects variable

\begin{tabular}{lllll}
\hline Variable & Levels & Estimate & SE & $P$-value \\
\hline Intercept & & 2.79 & 0.06 & $<0.001$ \\
Sampling month & February 2016 & Ref & - & - \\
& August 2016 & 0.39 & 0.06 & $<0.001$ \\
& October 2016 & 0.09 & 0.03 & 0.003 \\
& January 2017 & -0.13 & 0.03 & $<0.001$ \\
& April 2017 & -0.06 & 0.03 & 0.016 \\
Location & Aiguaviva & Ref & - & - \\
& Ordis & 0.06 & 0.10 & 0.563 \\
& Madremanya & -0.04 & 0.09 & 0.652 \\
& Vidreres & -0.03 & 0.10 & 0.783 \\
& Massanes & 0.05 & 0.08 & 0.533 \\
& Hostalnou de Bianya & -0.16 & 0.09 & 0.074 \\
& Montagut & 0.18 & 0.09 & 0.035 \\
Leropositivity & St. Esteve de Llémena & -0.10 & 0.10 & 0.287 \\
& Canet d'Adri & -0.05 & 0.10 & 0.641 \\
& St. Feliu de Guíxols & -0.19 & 0.14 & 0.173 \\
& Seropositive & -0.08 & 0.08 & 0.302 \\
& Riels i Viabrea & -0.06 & 0.08 & 0.399 \\
& Vilobí d'Onyar & - & - \\
& Seronegative & Ref & -03 & 0.032 \\
\hline
\end{tabular}

Abbreviation: SE standard error

a Level of significance of $P$-value $<0.05$ was used

canine host. It has presented high levels of correlation with SGH in both experimentally [25] and naturally bitten dogs $[16,17,26]$. Two apyrase proteins (rSP01B and rSP01) have also shown a good correlation with SGH [25]. However, in a study where these three recombinant proteins presented similarly high correlations with $\mathrm{SGH}$, rSP03B presented the lowest data dispersion and was considered a better option [16]. These results were confirmed in a field trial, where single rSP03B demonstrated a higher correlation coefficient with SGH than the combination of rSP03B with rSP01 [17].

A similar correlation between SGH and rSP03B to the one obtained in the present study has been observed before in Umbria region (central Italy) $\left(r_{S}=0.56\right.$; 95\% CI: $0.38-0.71, P<0.001 ; n=96$ ), in a screening study of dog exposure to $P$. perniciosus across European CanL endemic foci [26]. A possible reason for these discordant results may be the presence of other closely related phlebotomine species which could induce cross-reactivity with the SGH [22]. In some parts of Catalonia, P. perniciosus is sympatric with $P$. ariasi, also a proven vector of L. infantum [10]. Due to the close relationship between $P$. perniciosus and $P$. ariasi, both belonging to the subgenus Larroussius, it is 
expected that they share similar salivary antigens [37]. When comparing the percentage of seropositive dogs detected by both methods during the study, results for SGH are higher (49.43\%) than for rSP03B (28.98\%). Also, median results per sampling location show differences between SGH and rSP03B: in some cases, the trend between antigens is very similar (e.g. sera from Sant Feliu de Guíxols); in other cases, there is a recognizable peak in anti-SGH IgG, while anti-rSP03B IgG shows no change (e.g. sera from Madremanya). These differences can also be observed over time in the same location, with humoral responses to $\mathrm{SGH}$ and $\mathrm{rSP} 03 \mathrm{~B}$ peaking in different months along the transmission season (e.g. Canet d'Adri). We may hypothesize that SGH, because it contains more proteins than the single-antigen rSP03B, will more likely cross-react with antibodies against $P$. ariasi, inducing a stronger unspecific reaction to this vector species. It would also mean that the prevalence of sand fly species responsible for $L$. infantum transmission in the province varies according to the location, and possibly in the same location throughout the transmission season, for which it would be interesting to perform further entomological studies in the region.

Correlation indexes between levels of antibodies against both salivary antigens and $L$. infantum infection were low [SGH: $r_{S}=0.27$ (95\% CI: 0.19-0.35, $P<0.001$ ); rSP03B: $r_{S}$ $=0.25$ (95\% CI: 0.18-0.32, $P<0.001)$ ]. Similar low correlations have been described before between sand fly bites and human visceral leishmaniasis (VL), while stronger correlations are reported between human cutaneous leishmaniasis $(\mathrm{CL})$ and recent vector exposure (reviewed in [23]). This can be explained by VL's longer incubation period and/or the differences in host immune responses to cutaneous and visceral infection [38]. Results from some studies in human populations also suggest that the repeated contact with non-infected sand flies could be correlated with markers of protection for VL [39]. Partial protection against $L$. major, an agent of $\mathrm{CL}$, has also been achieved in immunized mice by the bites of uninfected sand flies [40]. However, another study with BALB/c mice demonstrated that this type of immunity is limited to short-term exposure and questioned the efficacy of sand fly saliva-induced protection against Leishmania infection in CL endemic areas [41]. CanL follows a pattern which is more similar to VL than to CL, therefore a low correlation between humoral responses to sand fly saliva and Leishmania would be expected [15]. However, results of the multilevel linear regression model show a positive and statistically significant relationship between $P$. perniciosus bites and a seropositive status for $L$. infantum, both for SGH and rSP03B. Similar results have been described in other longitudinal field studies on both canine anti-P. perniciosus and anti-L. longipalpis IgG dynamics [17, 34]. Unlike cross-sectional surveys, longitudinal studies are able to detect the relationship between a higher number of sand fly bites at a given time point and a subsequent $L$. infantum infection. Therefore, this type of study is likely to better explain the relationship between these two events, which can take place several months apart.

\section{Conclusions}

The results of this study confirmed the applicability of both anti-P. perniciosus SGH and rSP03B IgG as markers of exposure to $L$. infantum vectors in natural dog populations from an endemic area. Canine humoral response to both antigens is compatible with the annual sand fly activity dynamics expected for the region. Significantly lower IgG levels were observed during the non-transmission season; despite the repeated exposure to sand flies during the summer months, there is a return to basal IgG levels in these dog populations during the winter. The comparative performance of SGH and rSP03B showed a moderate correlation, which might be explained by the occurrence of cross-reactions of SGH with other closely related sympatric sand flies. Further longitudinal studies in natural canine populations from endemic areas, together with entomological studies, should be carried out in order to corroborate this hypothesis. Nevertheless, both antigens are expected to detect only vectors of $L$. infantum, confirming their suitability for host-vector-parasite studies. Finally, the overall results support the CanL endemicity status for the study region, which had already been suggested by previous studies [33].

\section{Methods}

\section{Experimental design}

The study included a heterogeneous population of 176 dogs distributed by 12 locations in Girona Province (Catalonia, northeast of Spain), an area endemic for CanL [33]. These dogs were enrolled in a canine leishmaniosis vaccine field trial, but no statistically significant differences in L. infantum infection between groups were observed either during or at the end of the trial. These were all owned dogs, used mainly for hunting, but some breeding and racing individuals were also included. All animals were kept in large packs in open-air facilities, mostly in rural and periurban areas. Furthermore, no specific anti-sand fly insecticide treatments were applied, providing conditions for dog exposure to the vector. Dog density per study location varied between 4-23. The dogs were followed from February 2016 to April 2017 and blood samples, obtained by venepuncture and placed in $5 \mathrm{ml}$ EDTA tubes, were collected at 5 pre-determined time points (Table 1). Plasma was obtained and stored at $-40{ }^{\circ} \mathrm{C}$ until processing.

\section{Sand flies and salivary proteins}

A colony of P. perniciosus was reared under standard conditions as described previously [42]. Salivary glands were 
dissected from 4-6 day-old females, pooled at a concentration of 1 salivary gland per $1 \mu \mathrm{l}$ of $20 \mathrm{mM}$ Tris buffer with $150 \mathrm{mM} \mathrm{NaCl}$ and stored at $-80^{\circ} \mathrm{C}$. The P. perniciosus 43 $\mathrm{kDa}$ yellow-related recombinant protein (rSP03B, Genbank accn. DQ150622) was obtained from Apronex s.r.o. (Prague, Czech Republic) and quantified by the Lowry method (Bio-Rad, Hercules, California, USA) following the manufacturer's protocol.

\section{Serological detection of dog exposure to sand flies}

Anti- $P$. perniciosus IgG was measured by an in-house enzyme-linked immunosorbent assay (ELISA) as described previously [17]. All samples from a single dog were processed in the same plate. Briefly, microtiter plates were coated either with salivary gland homogenate (SGH) (40 ng per well, equivalent to 0.2 salivary gland) or with rSP03B $(5 \mu \mathrm{g} / \mathrm{ml})$ in $20 \mathrm{mM}$ carbonate-bicarbonate buffer (pH 9.5) and incubated overnight at $4{ }^{\circ} \mathrm{C}$. Plates were then blocked with $6 \%(\mathrm{w} / \mathrm{v})$ low fat dry milk in PBS with $0.05 \%$ Tween 20 (PBS-Tw). Canine plasma were diluted 1:200 for SGH and 1:100 for rSP03B in 2\% (w/v) low fat dry milk/PBS-Tw. Secondary antibodies (anti-dog IgG, Bethyl laboratories) were diluted 1:9000 in PBS-Tw. The reaction was stopped with $10 \% \mathrm{H}_{2} \mathrm{SO}_{4}$ and absorbance was measured at $492 \mathrm{~nm}$ using a Tecan Infinite M200 microplate reader (Tecan, Männedorf, Switzerland). Each sample was tested in duplicate and positive and negative controls were included in each plate. To account for the variability between plates, sample OD readings were normalized by dividing them by the mean OD of positive controls run in the same plate [43]. The normalized OD values were multiplied by 100 . Positivity cut-offs were calculated as the mean plus 3 standard deviations from 14 dog samples from a non-endemic area.

\section{Serological detection of $L$. infantum infection}

All samples were tested for the presence of IgG against $L$. infantum through an in-house enzyme-linked immunosorbent assay (ELISA), using a technique described previously [44, 45]. Again, serial samples from a single dog were tested in parallel on the same plate. Briefly, dog plasma samples diluted at 1:400 were incubated in titration plates (Costar ${ }^{\circ}$ Corning $^{\circ}$, New York, USA) previously coated with sonicated whole promastigotes at a protein concentration of $20 \mu \mathrm{g} / \mathrm{ml}$ in $0.05 \mathrm{M}$ carbonate buffer at $\mathrm{pH}$ 9.6. Protein A peroxidase (1:30,000, Sigma-Aldrich ${ }^{\circ}$, St. Louis, Missouri, USA) was used as conjugate and reactions were stopped with $\mathrm{H}_{2} \mathrm{SO}_{4} 3 \mathrm{M}$ when a pre-determined calibrator control serum reached an optical density of 450 at $450 \mathrm{~nm}$. Sample optical densities were read at $492 \mathrm{~nm}$. All samples were run in duplicate and the calibrator, positive and negative sera were included in all plates. Results were expressed in standard units $(\mathrm{U})$ compared to a calibrator control sample set arbitrarily at $100 \mathrm{U}$. The positivity cut-off was established at $24 \mathrm{U}$.

\section{Statistical analysis}

Statistical analyses were performed using $\mathrm{R}$ software (http://cran.r-project.org/) and Stata 15 software (StataCorp LP, College Station, TX, USA).

Correlations between IgG responses to $P$. perniciosus SGH and rSP03B and between each one of the salivary antigens and anti- $L$. infantum IgG levels were tested by the Spearman rank correlation test. Median OD values between time points were compared using the Wilcoxon signed rank sum test.

The relationship between anti-SGH and anti-rSP03B antibodies and sampling month, L. infantum infection status and location was tested by fitting multilevel linear regression models, taking into account the correlation between repeated measures of the same dogs over time. In the models, log-transformed anti-saliva or rSP03B normalized OD values were considered as continuous dependent variables and sampling month, $L$. infantum infection and location as categorical predictor variables. In order to assess variations in OD between the first sampling month and those following, "February 2016" was set as reference level for this variable. Likewise, the locality with the lowest median OD ("Aiguaviva") was considered to be the reference for the variable location. Finally, "seronegative" was set as the reference level for the variable $L$. infantum infection. The random component included dog and time to allow for variation at the intercept (between dogs) and the slope (over time). The inclusion of "dog" as a random effects variable significantly improved both models, with a between dog variance of $48 \%$ for SGH and of $47 \%$ for the rSP03B model. A $P$-value of $<0.05$ was considered to indicate statistical significance.

\section{Abbreviations \\ CanL: Canine leishmaniosis: CL: Human cutaneous leishmaniasis: \\ ELISA: Enzyme-linked immunosorbent assay; IgG: Immunoglobulin G; OD: Optical density; rSP03B: 43 kDa yellow-related recombinant protein; SGH: Salivary gland homogenate; VL: Human visceral leishmaniasis}

\section{Acknowledgements}

We thank all dog owners, for providing us access to their dogs. We also thank the reviewers for their helpful suggestions and comments, and Trelawny BondTaylor for language revision and editing of the final manuscript.

\section{Funding}

This project received funding from the European Union's Horizon 2020 research and innovation programme under Marie Sklodowska-Curie grant agreement $n^{\circ} 642609$. The study was also partially supported by ERD Funds, project CePaViP (CZ.02.1.01/0.0/0.0/16_019/0000759), Research Centre UNCE (project 204072), Departament d'Universitats, Recerca i Societat de la Informació de la Generalitat de Catalunya (grant 2014SGR026), Plan Nacional de I+D+i and Instituto de Salud Carlos III, Subdirección General de Redes y Centros de Investigación Cooperativa, Ministerio de Economía y Competividad, Spanish Network for Research in Infectious Diseases (grant REIPI RD12/0015); co-financed by European Development Regional Fund (ERDF) "A way to achieve Europe". MG belongs to RICET, a Tropical Disease Cooperative Research Network in Spain (grant RD12/0018/0010). ISGlobal is a member of the CERCA 
Programme, Generalitat de Catalunya. The supporters had no role in study design, data collection and analysis, decision to publish, or preparation of the manuscript.

\section{Availability of data and materials}

The datasets used and/or analysed during the current study are available from the corresponding authors upon reasonable request.

\section{Authors' contributions}

RV, LW, JC, PV and MG designed the study; RV, ED and MG performed the fieldwork; RV, TS and LW performed the lab work; TS and RV analysed and interpreted the data; RV and MG wrote the manuscript. All authors read and approved the final manuscript.

\section{Ethics approval and consent to participate}

The research protocol was submitted to the Ethics Committee on Animal Experimentation (CEEA) of University of Barcelona, which considered that an ethical approval was not required for this study. The project was also submitted to and approved by ISGlobal Internal Scientific Committee (ISC). All dog owners were informed about the research protocol and signed an informed consent allowing for sample and data collection.

\section{Consent for publication}

Not applicable.

\section{Competing interests}

The authors declare that they have no competing interests.

\section{Publisher's Note}

Springer Nature remains neutral with regard to jurisdictional claims in published maps and institutional affiliations.

\section{Author details}

'ISGlobal, Hospital Clínic - Universitat de Barcelona, Barcelona, Spain. ${ }^{2}$ Secció de Parasitologia, Departament de Biologia, Sanitat i Medi Ambient, Facultat de Farmàcia i Ciències de l'Alimentació, Universitat de Barcelona, Barcelona Spain. ${ }^{3}$ Department of Parasitology, Faculty of Science, Charles University, Praque, Czech Republic. ${ }^{4}$ Hospital Veterinari Canis, Girona, Spain.

\section{Received: 20 June 2018 Accepted: 24 September 2018}

\section{Published online: 11 October 2018}

\section{References}

1. WHO Expert Committee on the Control of the Leishmaniases \& World Health Organization. Control of the leishmaniases: report of a meeting of the WHO Expert Commitee on the Control of Leishmaniases, Geneva, 22-26 March 2010. Geneva: World Health Organization. http://www.who.int/iris/ handle/10665/44412. Accessed 15 Mar 2018

2. Dantas-Torres F. Canine leishmaniosis in South America. Parasit Vectors. 2009;2(Suppl. 1):S1

3. Gállego M. Zoonosis emergentes por patógenos parásitos: las leishmaniosis. Rev Sci Tech. (OIE). 2004;23:661-76.

4. Franco AO, Davies CR, Mylne A, Dedet J-P, Gállego M, Ballart C, et al. Predicting the distribution of canine leishmaniasis in western Europe based on environmental variables. Parasitology. 2011;138:1878-91.

5. Baneth G, Koutinas AF, Solano-Gallego L, Bourdeau P, Ferrer L. Canine leishmaniosis - new concepts and insights on an expanding zoonosis: Part one. Trends Parasitol. 2008;24:325-30.

6. Borja LS, de Sousa OMF, Solcà MDS, Bastos LA, Bordoni M, Magalhães JT, et al. Parasite load in the blood and skin of dogs naturally infected by Leishmania infantum is correlated with their capacity to infect sand fly vectors. Vet Parasitol. 2016:229:110-7.

7. Molina R, Amela C, Nieto J, San-Andrés M, González F, Castillo JA, et al. Infectivity of dogs naturally infected with Leishmania infantum to colonized Phlebotomus perniciosus. Trans R Soc Trop Med Hyg. 1994;88:491-3.

8. Alten B, Maia C, Afonso MO, Campino L, Jiménez M, González E, et al. Seasonal dynamics of phlebotomine sand fly species proven vectors of Mediterranean leishmaniasis caused by Leishmania infantum. PLoS Negl Trop Dis. 2016;10:e0004458.

9. Rioux JA, Guilvard E, Gállego J, Moreno G, Pratlong F, Portús M, et al. Intervention simultanée de Phlebotomus ariasi Tonnoir, 1921 et $P$. perniciosus Newstead, 1911 dans un même foyer. Infestations par deux zymodemes syntopiques. A propos d'une enquête en Catalogne (Espagne). In: Leishmania: Taxonomie et Phylogenèse Applications Écoépidémiologiques. Montpellier: IMEEE; 1986. p. 439-44.

10. Guilvard E, Gállego M, Moreno G, Fisa R, Rispail P, Pratlong F, et al. Infestation naturelle de Phlebotomus ariasi et Phlebotomus perniciosus (Diptera - Psychodidae) par Leishmania infantum (KinetoplastidaTrypanosomatidae) en Catalogne (Espagne). Parasite. 1996;3:191-2.

11. Ballart C, Guerrero I, Castells X, Barón S, Castillejo S, Alcover MM, et al. Importance of individual analysis of environmental and climatic factors affecting the density of Leishmania vectors living in the same geographical area: the example of Phlebotomus ariasi and P. perniciosus in northeast Spain. Geospat Health. 2014;8:389-403.

12. Aransay AM, Testa JM, Morillas-Marquez F, Lucientes J, Ready PD. Distribution of sandfly species in relation to canine leishmaniasis from the Ebro Valley to Valencia, northeastern Spain. Parasitol Res. 2004:94:416-20.

13. Sáez VD, Morillas-Márquez F, Merino-Espinosa G, Corpas-López V, Morales-Yuste M, Pesson B, et al. Phlebotomus langeroni Nitzulescu (Diptera, Psychodidae) a new vector for Leishmania infantum in Europe. Parasitol Res. 2018;117:1105-13.

14. Rohousova I, Ozensoy S, Ozbel Y, Volf P. Detection of species-specific antibody response of humans and mice bitten by sand flies. Parasitology. 2005;130:493-9.

15. Vlkova M, Rohousova I, Drahota J, Stanneck D, Kruedewagen EM, Mencke N, et al. Canine antibody response to Phlebotomus perniciosus bites negatively correlates with the risk of Leishmania infantum transmission. PLoS Negl Trop Dis. 2011;5:e1344.

16. Martín-Martín I, Molina R, Rohoušová I, Drahota J, Volf P, Jiménez M. High levels of anti-Phlebotomus perniciosus saliva antibodies in different vertebrate hosts from the re-emerging leishmaniosis focus in Madrid, Spain. Vet Parasitol. 2014:202:207-16.

17. Kostalova T, Lestinova T, Sumova P, Vlkova M, Rohousova I, Berriatua E, et al. Canine antibodies against salivary recombinant proteins of Phlebotomus perniciosus: a longitudinal study in an endemic focus of canine leishmaniasis. PLoS Negl Trop Dis. 2015;9:e0003855.

18. Marzouki S, Ben Ahmed M, Boussoffara T, Abdeladhim M, Ben Aleya-Bouafif $\mathrm{N}$, Namane A, et al. Characterization of the antibody response to the saliva of Phlebotomus papatasi in people living in endemic areas of cutaneous leishmaniasis. Am J Trop Med Hyg. 2011;84:653-61.

19. Carvalho AM, Cristal JR, Muniz AC, Carvalho LP, Gomes R, Miranda JC, et al. Interleukin 10-dominant immune response and increased risk of cutaneous leishmaniasis after natural exposure to Lutzomyia intermedia sand flies. J Infect Dis. 2015;212:157-65.

20. Gidwani K, Picado A, Rijal S, Singh SP, Roy L, Volfova V, et al. Serological markers of sand fly exposure to evaluate insecticidal nets against visceral leishmaniasis in India and Nepal: a cluster-randomized trial. PLoS Negl Trop Dis. 2011;5:e1296.

21. Clements MF, Gidwani K, Kumar R, Hostomska J, Dinesh DS, Kumar V, et al. Measurement of recent exposure to Phlebotomus argentipes, the vector of Indian visceral leishmaniasis, by using human antibody responses to sand fly saliva. Am J Trop Med Hyg. 2010;82:801-7.

22. Volf $\mathrm{P}$, Rohoušova I. Species-specific antigens in salivary glands of phlebotomine sandflies. Parasitology. 2001;122:37-41.

23. Lestinova $T$, Rohousova I, Sima $\mathrm{M}$, de Oliveira $\mathrm{Cl}$, Volf $\mathrm{P}$. Insights into the sand fly saliva: blood-feeding and immune interactions between sand flies, hosts, and Leishmania. PLoS Negl Trop Dis. 2017:11:e0005600.

24. Lestinova T, Vlkova M, Votypka J, Volf P, Rohousova I. Phlebotomus papatasi exposure cross-protects mice against Leishmania major co-inoculated with Phlebotomus duboscai salivary gland homogenate. Acta Trop. 2015;144:9-18.

25. Drahota J, Martin-Martin I, Sumova P, Rohousova I, Jimenez M, Molina R, et al. Recombinant antigens from Phlebotomus perniciosus saliva as markers of canine exposure to visceral leishmaniases vector. PLoS Negl Trop Dis. 2014; $8 \cdot e 2597$.

26. Kostalova $T$, Lestinova $T$, Maia $C$, Sumova $P$, Vlkova $M$, Willen $L$, et al. The recombinant protein $\mathrm{rSPO3B}$ is a valid antigen for screening dog exposure to Phlebotomus perniciosus across foci of canine leishmaniasis. Med Vet Entomol. 2016:31:88-93.

27. Hostomska J, Rohousova I, Volfova V, Stanneck D, Mencke N, Volf P. Kinetics of canine antibody response to saliva of the sand fly Lutzomyia longipalpis. Vector Borne Zoonotic Dis. 2008;8:443-50

28. Sima M, Ferencova B, Warburg A, Rohousova I, Volf P. Recombinant salivary proteins of Phlebotomus orientalis are suitable antigens to measure exposure of domestic animals to sand fly bites. PLoS Negl Trop Dis. 2016:10: e0004553. 
29. Rohousova I, Talmi-Frank D, Kostalova T, Polanska N, Lestinova T, Kassahun A, et al. Exposure to Leishmania spp. and sand flies in domestic animals in northwestern Ethiopia. Parasit Vectors. 2015;8:360.

30. Morillas Marquez F, Guevara Benitez DC, Ubeda Ontiveros JM, Gonzalez Castro J. Fluctuations annuelles des populations de Phlébotomes (Diptera, Phlebotomidae) dans la province de Grenade (Espagne). Ann Parasitol Hum Comp. 1983;58:625-32.

31. Gálvez R, Descalzo MA, Miró G, Jiménez MI, Martín O, Dos Santos-Brandao F, et al. Seasonal trends and spatial relations between environmental/ meteorological factors and leishmaniosis sand fly vector abundances in central Spain. Acta Trop. 2010;115:95-102.

32. González E, Jiménez M, Hernández S, Martín-Martín I, Molina R. Phlebotomine sand fly survey in the focus of leishmaniasis in Madrid, Spain (2012-2014): seasonal dynamics, Leishmania infantum infection rates and blood meal preferences. Parasit Vectors. 2017:10:368.

33. Lladró S, Picado A, Ballart C, Portús M, Gállego M. Management, prevention and treatment of canine leishmaniosis in north-eastern Spain: an online questionnaire-based survey in the province of Girona with special emphasis on new preventive methods (CaniLeish vaccine and domperidone). Vet Rec 2017;180:47.

34. Quinnell RJ, Soremekun S, Bates PA, Rogers ME, Garcez LM, Courtenay O. Antibody response to sand fly saliva is a marker of transmission intensity but not disease progression in dogs naturally infected with Leishmania infantum. Parasit Vectors. 2018;11:7.

35. Marzouki S, Kammoun-Rebai W, Bettaieb J, Abdeladhim M, Hadj Kacem S, Abdelkader R. Validation of recombinant salivary protein PpSP32 as a suitable marker of human exposure to Phlebotomus papatasi, the vector of Leishmania major in Tunisia. PLoS Negl Trop Dis. 2015;9:e0003991.

36. Souza AP, Andrade BB, Aquino D, Entringer P, Miranda JC, Alcantara R, et al. Using recombinant proteins from Lutzomyia longipalpis saliva to estimate human vector exposure in visceral leishmaniasis endemic areas. PLoS Negl Trop Dis. 2010;4:e649.

37. Anderson JM, Oliveira F, Kamhawi S, Mans BJ, Reynoso D, Seitz AE, et al. Comparative salivary gland transcriptomics of sandfly vectors of visceral leishmaniasis. BMC Genomics. 2006;7:52

38. Kedzierski L, Evans KJ. Immune responses during cutaneous and visceral leishmaniasis. Parasitology. 2014;141:1544-62.

39. Andrade BB, Teixeira CR. Biomarkers for exposure to sand flies bites as tools to aid control of leishmaniasis. Front Immunol. 2012;3:121.

40. Kamhawi S, Belkaid Y, Modi G, Rowton E, Sacks D. Protection against cutaneous leishmaniasis resulting from bites of uninfected sand flies. Science. 2000:290:1351-4.

41. Rohoušová I, Hostomská J, Vlková M, Kobets T, Lipoldová M, Volf P. The protective effect against Leishmania infection conferred by sand fly bites is limited to short-term exposure. Int J Parasitol. 2011:41:481-5.

42. Volf $\mathrm{P}$, Volfova $\mathrm{V}$. Establishment and maintenance of sand fly colonies. J Vector Ecol. 2011;36:S1-9.

43. Sanchez J, Dohoo IR, Markham F, Leslie K, Conboy G. Evaluation of the repeatability of a crude adult indirect Ostertagia ostertagi ELISA and methods of expressing test results. Vet Parasitol. 2002;109:75-90.

44. Ballart C, Alcover MM, Picado A, Nieto J, Castillejo S, Portús M, et al. First survey on canine leishmaniasis in a non classical area of the disease in Spain (Lleida, Catalonia) based on a veterinary questionnaire and a crosssectional study. Prev Vet Med. 2013;109:116-27.

45. Riera C, Valladares JE, Gállego M, Aisa MJ, Castillejo S, Fisa R, et al. Serological and parasitological follow-up in dogs experimentally infected with Leishmania infantum and treated with meglumine antimoniate. Vet Parasitol. 1999;84:33-47.

\section{Ready to submit your research? Choose BMC and benefit from:}

- fast, convenient online submission

- thorough peer review by experienced researchers in your field

- rapid publication on acceptance

- support for research data, including large and complex data types

- gold Open Access which fosters wider collaboration and increased citations

- maximum visibility for your research: over $100 \mathrm{M}$ website views per year

At $\mathrm{BMC}$, research is always in progress.

Learn more biomedcentral.com/submissions 